\title{
Linx
}

Revue des linguistes de l'université Paris X Nanterre

12 | 2002

"Comme la lettre dit la vie »

\section{Du métatextuel en poésie}

Jean-François Jeandillou

\section{Q OpenEdition}

\section{Journals}

Édition électronique

URL : http://journals.openedition.org/linx/1288

DOI : 10.4000/linx.1288

ISSN : 2118-9692

\section{Éditeur}

Presses universitaires de Paris Nanterre

\section{Édition imprimée}

Date de publication : 1 octobre 2002

Pagination : 101-105

ISSN : 0246-8743

\section{Référence électronique}

Jean-François Jeandillou, « Du métatextuel en poésie », Linx [En ligne], 12 | 2002, mis en ligne le 10 octobre 2012, consulté le 06 mai 2019. URL : http://journals.openedition.org/linx/1288 ; DOI :

10.4000/linx.1288

Ce document a été généré automatiquement le 6 mai 2019.

Département de Sciences du langage, Université Paris Ouest 


\title{
Du métatextuel en poésie
}

\author{
Jean-François Jeandillou
}

1 L'agrégation a son secret, le devoir en temps limité a son mystère. Nous présentons ciaprès un document naguère réalisé dans les conditions, ô combien rigoureuses, d'une épreuve dite de "concours blanc ». L'auteur, qui a bien voulu laisser divulguer sa copie tout en conservant l'anonymat, n'eut alors pas le loisir d'en achever la rédaction, ni même de mener à terme sa réflexion ; aussi est-ce sous la forme de notes - abrégées, plus ou moins allusives et parfois déconcertantes - que se donnent à lire ces quelques pages initialement conçues comme une «étude stylistique » du poème intitulé, dans les Stèles de Victor Segalen, « Retombée ${ }^{1}$ ». Sans autrement insister sur les raisons qui nous ont incité à reproduire telle quelle cette ébauche (avec ses maladresses, ses imperfections mais aussi sa rare pertinence), nous tenons cependant à préciser que la signataire connut un heureux succès, quelques mois plus tard, face au prestigieux jury qui, pour l'avoir lue puis entendue, inscrivit son nom sur la liste des lauréats et dans un rang de classement fort honorable.

2 C'est la septième des « Stèles du Milieu », exactement centrale (six avant, six après). Donc, position clé, au mitan du mitan. Cf. fin de la Préface :

Certaines, qui ne regardent ni le sud ni le nord, ni l'est ni l'occident, ni aucun des points interlopes, désignent le lieu par excellence, le milieu. Comme les dalles renversées ou les voûtes gravées dans la face invisible, elles proposent leurs signes à la terre qu'elles pressent d'un sceau. Ce sont les décrets d'un autre empire, et singulier. On les subit ou on les récuse, sans commentaires ni gloses inutiles, d'ailleurs sans confronter jamais le texte véritable : seulement les empreintes qu'on lui dérobe. (nous soulignons)

D'où amphibologie du titre, qui, référant à l'architecture, laisse aussi attendre des retombées (comme nous l'avons montré dans notre étude lexicologique du mot, au début de ce devoir) provenant « d'un autre empire ». Toute l'organisa ${ }^{\circ}$ rhétorique du poème, fondée sur la description d'une pagode et de sa toiture, implique ainsi, grâce au jeu des 
comparaisons, la réinterprétation à la fois allégorique et déceptive d'un «texte véritable » qui demeure caché.

4 Après avoir analysé structure quasi géométrique et dispositif énonciatif de ce poème où forte prégnance du JE, examiner, à travers le matériel lexical, les ambiguïtés mêmes de l'évocation.

\section{L'écho d'une voix anonyme}

\section{1) Quatre séquences symétriques deux à deux}

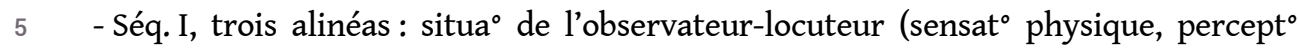
visuelle, disposi ${ }^{\circ}$ mentale), avec récurrence du JE + présent.

6 - Séq. II, trois alinéas : dispari ${ }^{\circ}$ du JE, sélection d'une partie minimale et essentielle de l'édifice, selon trois dénominations successives: al. 1, «les Angles»; al. 2, «ces quatre cornes » avec question; al. 3, « les quatre coins » avec réponse provisoire à question.

7 - Séq. III : dans chacun des deux alinéas, deux quest ${ }^{\circ}$ sur les liens d'abord, l'au-delà ensuite.

8 - Séq. IV, deux al. : dernière quest ${ }^{\circ}$ suivie de non-réponse, puis explica ${ }^{\circ}$ matérialiste de ces éléments d'architecture, avec réappari ${ }^{\circ}$ du JE in extremis.

Donc composit ${ }^{\circ}$ très serrée, les séq. sont numériquement égales aux quatre chevrons $\rightarrow$ iconicité du texte. Nombreuses correspondances formelles : les JE, les quest ${ }^{\circ}$, les disposit $^{\circ}$ d'alinéas, les démonstratifs déictiques (1.12-24).

10 Mais il ne s'agit là que des apparences, illusoires et vaines. Celles-ci doivent ê dépassées.

Cf. « Maïeutique » (p. 144, nous soulignons) :

Faire son plan: construire le Palais factice, prêt à conserver cadres et murs si le Germe s'y trouve mal à son aise.

Ne pas trop « penser » : la pensée : anecdote de l'esprit. Mais, le dos rond, ramassé, hérissé, farouche,

Bondir sur le Germe, dès qu'il est né, le secouer et l'étirer, le serrer et l'écrouir pour connaître s'il est, ou non, digne de la densité des Mots.

11 Le Robert : écrouir, pratiquer l'écrouissage : Techn. Opération consistant à travailler (en le frappant, laminant, étirant) un métal à une température inférieure à sa température de recuit ; effet ainsi obtenu (résistance à la déformation).

\section{2) Défaut d'embrayage}

- Pron sujet $1^{\mathrm{e}}$ pers., indice du locuteur :

- alinéa 1, quatre phrases minimales : JE + vb + cplmt.

- al. 2 : trois phr semblables, mais la deuxième comporte expansion de 4 cod.

- al. 3: une seule phrase complexe, avec coord de deux propos ${ }^{\circ}$ dont JE est sujet, + occurrce de $\mathrm{ME}$ en posit $^{\circ}$ d'attaque.

$16-1.19$, retour ultime de ce $\mathrm{JE}+\mathrm{vb}$ de percept $^{\circ}$ au présent. 
17 Mais JE reste anonyme : qui parle? voix sans origine, sans identité, qu'on ne saurait assimiler à celle du poète/auteur, d'autant qu'il s'agit censémt d'un écrit stélaire, gravé dans la pierre... doter d'aspect itératif (présent d'habitude, $n$ fois). D'ailleurs, les autres prst ccernant les chevrons décrits ont valeur étendue, qui correspond à tout instant où le référent est appréhendable. parvis inverse, DU faîte, DU toit, et à la fin $L E$ grossier piquet... Le processus proprement anaphorique est ici bloqué par l'absence d'antécédent explicite : exophore mémorielle? ou bien réf situationnelle non saturée (et vague) ? Dans la mesure où le réf exact n'est jamais nommé ni défini, le lecteur doit le recstituer à partir des indices fournis. Dès lors, les art déf présupposent connu le réf, et $\mathrm{y}$ renvoient par anaphore associative (synecdoque, de la partie au tout).

\section{3) Retombée des mots, retombée des phrases}


Je FrAP(pe) les DAL(les). // J’en éprou(ve) la solidité. // J'en écou(te) la sonorité. // Le me sens FER $\underline{m}(e)$ et satisfaIt.

J'embras(se) les colon(nes). // Je mesur(e) leur JET, / la portéE, / le nombr(e) et la plantation. // Je me sens clos et satisfait.

- Séq. II de retard.

- Séq. III levÉEs? interrogat).

- Séq. IV

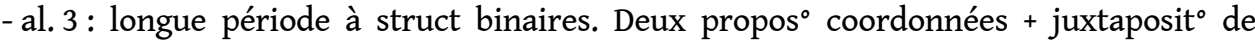
cplmts doubles :

(Me renversant,) cou tendu, / nuque douloureuse

(lourd habit cérémonieux,) aux plis carrés, / à la forte charpente

NB : «cou tendu », trait descriptif déjà signalé dans la Préface, à propos de la «tortue » chinoise. Sous-phrase, cstruct $^{\circ}$ absolue détachée, de type thème + prédicat attributif, sans déterminant... (cf. Le Goffic)

- al. 1 : ccst ppst, avec apposit (" paisible horizon terrestre »), suivi du GN thématique, avec trois épithètes détachées : voici les Angles/ acérés / griffus et cornus. La ponctua ${ }^{\circ}$ forte $\mathrm{du}$ tiret n'est pas syntaxique mais prosodique : pause, silence dilatoire, effet d'attente et

Homéotéleute en /-y/, allitéra ${ }^{\circ}$ en /R/, et aussi Mûri coMMe un Manteau des Moissons.

- al. 2 : questions avec inversion simple ou complexe du sujet, et disposition en chiasme :

CES quatre cornes, QUI menacent-elles...

QUE découvrent CES quatre doigts...

Sonorités récurrentes ds $3^{\mathrm{e}}$ quest $^{\circ}$ : Font-Ils sIgne QU'Il Y A IA-haut QQun QuI regArde

- al. 3 : phrase complexe, quatre occurrences de QUATRE $(1.12,13,15,15)$ : composit $^{\circ}$ « orchestique » de la stèle.

- al. 1 : quatre quest ${ }^{\circ}$, avec triple répét ${ }^{\circ}$ du morphème introducteur (6 /6 / 12) :

$\underline{A}$ quels piliERs du CIEL, à quels poTEAUX $\underline{\text { du }}$ MOND(e), à quelles HAMpes dix mille FoIs é

- al. 2: thématisa ${ }^{\circ}$ du cod, avec deux ppés détachés. Allitération en $/ \mathrm{p} /$ et volume syllabique croissant $(3 / 5 / 3+5)$ :

cet esPAC(e), / crevÉ par les POINT(es), / pénéTRÉ des neuf firmaMENTS,

Polyptote : liens-se lier (+ homophonie piliers)

$\rightarrow$ Volume inverse décroissant dans $2^{\mathrm{e}}$ phrase: (6+5) / 5 / 3 (avec seulement pron

- al. 1 : reformulation : griffus-ces quatre doigts aux ongles longs (11-13) // ces quatre doigts courbés aux ongles longs.

Même rôle (cf. 1. 10) prosodique du tiret avant VOICI.

A nouveau quatre négat ${ }^{\circ}$, avec juxtaposit ${ }^{\circ}$ coordinative (polysyndète ET) : régularité des mesures 4 / 4 / $6 / 6(/+4)$

- al. 2 : prolongement (syntaxique) d'al. 1, mais nouvel énoncé, car changement d'alinéa, et séparat ${ }^{\circ}$ par ponctua ${ }^{\circ}$ forte. Phrase en accent circflexe (avec struct binaires) : 
Puisque sous chacun des chevrons volants, accusant sa corne, résolvant sa cambrure, /

j'aperçois le grossier piquet terrestre qui le soutient et qui l'explique.

53 Anagr. : volant / résolvant, piquet / explique. Et /p/-/l/-/k/, /E/-/i/ : aPerçois-Le-PiQuet-QuiLe-Qui-L'exPLiQue.

\section{Le « haut mystère » en questions}

\section{1) De l'hypotypose à la figuration}

- Champ lexical de perception sensorielle : éléments du bâtimt et aussi du corps, qui encadrent le txt au début

- dalles, colonnes, planta ${ }^{\circ}$, charpente / cou, nuque, épaules, et ts les vb...

- faîte (voir laPréface), bords du toit,

et à la fin (après envolées interprétatives, qui retombent à plat) : chevrons, piquet terrestre L'architecture chinoise [...] amplifie et multiplie les toits [...]. De là l'emploi des tuiles noires formant des rainures profondes et de fortes côtes, qui, en haut laissant entre elles des jours, détachent et dégagent le faîte : amenuisé, fleuri, il découpe dans l'air lucide sa frise.

(P. Claudel, Connaissance de l'Est, p. 14)

- Métaphores Séq 2 sont toutes attestées dans la Préface du recueil :

- animalière : griffus et cornus, cornes, doigts aux ongles (aussi 1. 24).

- vestimentaire : lourd habit cérémonieux, manteau des moissons. Rapp cparant-cparé par similitude formelle du drapé : effet poétique (mais provisoire et déceptif) de l'image, puisque ce sont encore les ornemts du toit qui justifient métaphore.

- architecturale : coulant du faîte, paisible horizon terrestre, Tente originale (symbole plutôt qu'objet matériel, comme l'indique l'initiale majuscule), liens $(16,18)$, piliers, poteaux, hampes : reprend lex de base, mais en emploi figuré.

$61 \rightarrow$ isotopie terrestre (répété 1.9 et 19) / céleste: ciel (12, 19, avec majuscule, Ciel, représenta ${ }^{\circ}$ mystique, entité abstraite, quasi-divine...), là-ht, au-delà des nues (collectifs poétique abstrait), chevrons volants, parvis inverse.

62 Mouvement anagogique - du bas vers le haut (neuffirmaments, confins, Extrême, Grand-Vide ), du visible connu vers l'invisible énigmatique, du matériel tangible vers l'illumination absolue - mais suivi d'une retombée / chute : les comparaisons et métaphores sont dépourvues de fondement, la transcendance imperceptible n'a pas d'existence.

63 C'est précisémt cette absence de « répons » que vient pallier le poème lui-même, par élabora ${ }^{\circ}$ d'un discours mimétique de ce qu'il représente. Le texte poétique enveloppe sa propre allégorie.

\section{2) Le « Wen »}

64 «J'en écoute la sonorité»:quasi objet interne, tautologie... $\rightarrow$ c'est la sonorité incantatoire du poème qui, en l'occurrence, se fait entendre (cf. supra allitéra ${ }^{\circ}$, rythmes et assonances) 

l'inconnu, sans solution / clé de lecture analytique : espace structuré, libre d'accès mais néanmoins opaque et résistant («inquiétant», selon l'épigraphe chinoise). Comme l'« arrivant » que vient viser l'œil du ciel (dans la Préface), c'est celui qui croit regarder (je marche du regard // qqn qui regarde) qui est vu. intérieur dont ce poème, plus que le doublet symbolique, est à la fois l'analogon verbal et l'image écrite. Loin de se poser en apologie de l'ineffable, il révoque en doute l'ontologie de ce qui, ne se faisant pas connaître, ne peut être nommé. Voilà pourquoi les divers alinéas peuvent se comprendre en relation avec le texte saisi comme dispositif spéculaire: j'en écoute la sonorité, forte charpente, font-ils signe, la Tente originale, livrant avenue, ample hospitalité...

73 Paradoxale, sous ses dehors de haute spiritualité, cette poétique matérialiste n'appelle $d$ ' explication formelle que réflexive et/ou métatextuelle: «On les subit ou on les récuse, 
sans commentaires ni gloses inutiles, - d'ailleurs sans confronter jamais le texte véritable : seulement les empreintes qu'on lui dérobe.»

\section{NOTES}

1. L'édition au programme était celle d'Henri Bouillet, Paris, Robert Laffont, coll. «Bouquins ", 1995, p. 114.

\section{AUTEUR}

JEAN-FRANÇOIS JEANDILLOU

IUF, Paris 10 - Nanterre 